\title{
ENDOMORPHISMS OF THE COHOMOLOGY OF COMPLEX GRASSMANNIANS
}

BY

MICHAEL HOFFMAN

\begin{abstract}
For any complex Grassmann manifold $G$, we classify all endomorphisms of the rational cohomology ring of $G$ which are nonzero on dimension 2 . Some applications of this result are given.
\end{abstract}

1. Introduction. Let $G_{k, n}$ denote the complex Grassmann manifold of $k$-dimensional subspaces of $\mathbf{C}^{n+k}$. Then $G_{k, n}$ is a $k n$-dimensional complex manifold. In this paper we give a partial classification of endomorphisms of the cohomology ring $H^{*}\left(G_{k, n} ; \mathbf{Q}\right)$. This is useful as a first step toward classifying self-maps of $G_{k, n}$, and has several other consequences (see $§ 7$ ).

The cohomology ring $H^{*}\left(G_{k, n} ; \mathbf{Q}\right)$ is generated by elements $c_{1}, c_{2}, \ldots, c_{k}$, with $c_{i} \in H^{2 i}\left(G_{k, n}\right.$; Q). Since $c_{1}$ is the only generator in dimension 2, any endomorphism of $H^{*}\left(G_{k, n} ; \mathbf{Q}\right)$ sends $c_{1}$ to a multiple of itself. The main result of this paper is as follows.

THEOREM 1.1. Let $h$ be an endomorphism of $H^{*}\left(G_{k, n} ; \mathbf{Q}\right)$ with $h\left(c_{1}\right)=m c_{1}, m \neq 0$. Then if $k<n$,

$$
h\left(c_{i}\right)=m^{i} c_{i}, \quad 1 \leqslant i \leqslant k .
$$

If $k=n$, there is the additional possibility

$$
h\left(c_{i}\right)=(-m)^{i}\left(c^{-1}\right)_{i}, \quad 1 \leqslant i \leqslant k,
$$

where $\left(c^{-1}\right)_{i}$ is the 2i-dimensional part of the inverse of $c=1+c_{1}+\cdots+c_{k}$ in $H^{*}\left(G_{k, n} ; \mathbf{Q}\right)$.

RemarK. A Hermitian inner product on $\mathbf{C}^{n+k}$ gives a homeomorphism of $G_{k, n}$ onto $G_{n, k}$ by orthogonal complementation, so there is no loss of generality in considering only $G_{k, n}$ with $k \leqslant n$.

Theorem 1.1 includes a classification of automorphisms of $H^{*}\left(G_{k, n} ; \mathbf{Q}\right)$. Such a classification, for $k<n$, was first given in the unpublished work of Brewster [3], and Brewster's result is used in [4] to prove Theorem 1.1 for $k<n$. The proof given here draws heavily on [3], but is shorter and includes the case $k=n$. The main innovation is the use of the hard Lefschetz theorem.

Received by the editors November 30, 1982. A contributed talk based on this paper was presented to the winter meeting of the Canadian Mathematical Society on December 13, 1982.

1980 Mathematics Subject Classification. Primary 55S37; Secondary 14M15, 57T15.

Key words and phrases. Grassmann manifold, self-map, cohomology ring, endomorphism, hard Lefschetz theorem, Schubert calculus.

(C)1984 American Mathematical Society $0002-9947 / 84 \$ 1.00+\$ .25$ per page 
To complete the classification of endomorphisms of $H^{*}\left(G_{k, n} ; \mathbf{Q}\right)$, it suffices to describe those endomorphisms $h$ with $h\left(c_{1}\right)=0$. We make the following conjecture.

CONJECTURE 1.2. The only endomorphism $h$ of $H^{*}\left(G_{k, n} ; \mathbf{Q}\right)$ with $h\left(c_{1}\right)=0$ is the zero endomorphism.

If this conjecture is true, then the hypothesis " $m \neq 0$ " in Theorem 1.1 can be omitted. The conjecture is true if $n \geqslant 2 k^{2}-k-1$ or $k \leqslant 3$, as follows from [ 16 and 5] (except for the cases $n=k=2$ and $n=k=3$, which are easily checked).

Theorem 1.1 is proved in $\$ \S 3-6$, after a discussion of the cohomology ring $H^{*}\left(G_{k, n} ; \mathbf{Q}\right)$ in $\S 2$. Some applications are discussed briefly in $\$ 7$.

The results of this paper are part of the author's thesis [11] written under the supervision of F. P. Peterson. The author is indebted to him for encouragement and suggestions. Also important to this work were conversations of the author with Alain Lascoux and Richard Stanley. Finally, the author thanks Bill Homer for directing him to [5 and 3].

2. The cohomology ring. The following characterization of $H^{*}\left(G_{k, n} ; \mathbf{Q}\right)$ comes from results in [2].

THEOREM 2.1. Let $\gamma$ be the canonical $k$-plane bundle over $G_{k, n}$. Then if $c=1+c_{1}$ $+\cdots+c_{k}$ is the total Chern class of $\gamma$,

$$
H^{*}\left(G_{k, n} ; \mathbf{Q}\right)=\mathbf{Q}\left[c_{1}, \ldots, c_{k}\right] / I_{k, n},
$$

where $I_{k, n}$ is the ideal generated by $\left(c^{-1}\right)_{n+i}, 1 \leqslant i \leqslant k\left(\right.$ here $\left(c^{-1}\right)_{q}$ is the part of the formal inverse of $c$ in dimension $2 q$ ).

REMARK. $I_{k, n}$ contains all the elements $\left(c^{-1}\right)_{q}, q>n$, and in fact

$$
c^{-1}=1+\left(c^{-1}\right)_{1}+\cdots+\left(c^{-1}\right)_{n} \in H^{*}\left(G_{k, n} ; \mathbf{Q}\right)
$$

is the Chern class of the orthogonal complement bundle of $\gamma$.

It is well known that the universal Chern classes $c_{i} \in H^{*}\left(B U_{k} ; \mathbf{Q}\right)$ can be identified with the elementary symmetric functions

$$
e_{i}\left(t_{1}, \ldots, t_{k}\right)=\sum_{m_{1}<\cdots<m_{i}} t_{m_{1}} \cdots t_{m_{i}}
$$

in $\mathbf{Q}\left[t_{1}, \ldots, t_{k}\right]$, where each $t_{i}$ is in dimension 2. Since $H^{*}\left(G_{k, n} ; \mathbf{Q}\right)$ is a quotient of $H^{*}\left(B U_{k} ; \mathbf{Q}\right)=\mathbf{Q}\left[c_{1}, \ldots, c_{k}\right]$, we can regard $H^{*}\left(G_{k, n} ; \mathbf{Q}\right)$ as a quotient of the ring $\mathbf{Q}\left[t_{1}, \ldots, t_{k}\right]^{\Sigma_{k}}$ of symmetric functions in $k$ variables. To describe the ideal $I_{k, n}$ in this setting, we shall require some facts about the symmetric functions.

We first establish notation for partitions. A partition $\lambda$ of an integer $n \geqslant 0$ is a sequence $\lambda_{1} \geqslant \lambda_{2} \geqslant \cdots \geqslant \lambda_{l}>0$ of integers with $\lambda_{1}+\cdots+\lambda_{l}=n$. We call $\lambda_{1}$ the greatest part of $\lambda$, and $l(\lambda)=l$ the length of $\lambda$. We write $\lambda=\lambda_{1} \lambda_{2} \cdots \lambda_{1}$, and $|\lambda|=n$. Sometimes we shall use superscripts to indicate repetition, so $3^{2} 21$ means 3321 and not 921. Associated to $\lambda$ is its diagram, which is a left-justified array of boxes with $\lambda_{i}$ boxes in the $i$ th row. For example, $\lambda=3321$ has diagram as shown below. 


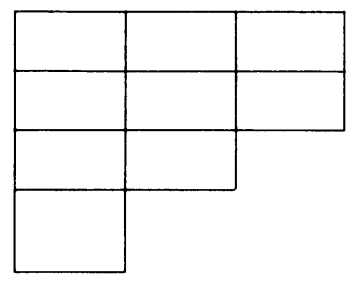

By reflecting the diagram of $\lambda$ through its main diagonal, we get the diagram of another partition $\lambda^{\prime}$ with $\left|\lambda^{\prime}\right|=|\lambda|$. Formally,

$$
\lambda_{p}^{\prime}=\text { order of }\left\{q \mid \lambda_{q} \geqslant p\right\} \text {. }
$$

The partition $\lambda^{\prime}$ is called the conjugate partition of $\lambda$ : conjugation is an involution exchanging greatest part and length.

Now we consider the ring $\Lambda_{k}=\mathbf{Q}\left[t_{1}, \ldots, t_{k}\right]^{\Sigma_{k}}$ of symmetric functions in $k$ variables. As an algebra, $\Lambda_{k}$ is generated by the elementary symmetric functions $e_{1}, \ldots, e_{k}$. Another set of symmetric functions $h_{1}, h_{2}, \ldots$ is defined by

$$
h_{i}=\sum_{p_{1}+\cdots+p_{k}=i} t_{1}^{p_{1}} t_{2}^{p_{2}} \cdots t_{k}^{p_{k}} .
$$

The $h_{i}$ are called symmetric functions. The following identity relates the complete and elementary symmetric functions $[15$, p. 14]:

$$
\left(1+e_{1}+\cdots+e_{k}\right)\left(1-h_{1}+h_{2}-\cdots\right)=1 .
$$

Another identity is proved in [15, p. 15].

Proposition 2.2. For any partition $\lambda=\lambda_{1} \lambda_{2} \cdots \lambda$, with $l \leqslant k$,

$$
\operatorname{det}\left(h_{\lambda_{p}-p+q}\right)_{1 \leqslant p, q \leqslant 1}=\operatorname{det}\left(e_{\lambda_{p}^{\prime}-p+q}\right)_{1 \leqslant p, q \leqslant \lambda_{1}} \text {. }
$$

The common value of both sides of (2) is called the Schur function (or S-function) associated to $\lambda$ and denoted $s_{\lambda}$. The $s_{\lambda}$ are symmetric functions, and in fact $\left\{s_{\lambda} \mid l(\lambda) \leqslant k\right\}$ is a basis for $\Lambda_{k}[15$, p. 24].

We are now in a position to describe $I_{k, n}$, hence $H^{*}\left(G_{k, n} ; \mathbf{Q}\right)$, in terms of symmetric functions. By (1), we see that identifying $c_{i}$ and $e_{i}$ with identifies $\left(c^{-1}\right)_{i}$ with $(-1)^{i} h_{i}$. Hence $I_{k, n}$, considered as an ideal in $\Lambda_{k}$, is generated by $h_{n+i}, \ldots, h_{n+k}$. This ideal contains all the $h_{i}, i>n$ (use (1)). So we have the following result.

THEOREM 2.3. There is an isomorphism $\Lambda_{k} /\left\{h_{1} \mid i>n\right\} \stackrel{\sim}{\rightarrow} H^{*}\left(G_{k, n} ; \mathbf{Q}\right)$ taking $e_{i}\left(t_{1}, \ldots, t_{k}\right)$ to $c_{i}$.

Now it is a classical result of algebraic geometry that $H^{*}\left(G_{k, n} ; \mathbf{Q}\right)$ has basis $\left\{\sigma_{\lambda} \mid \lambda_{1} \leqslant n, l(\lambda) \leqslant k\right\}$, where the elements $\sigma_{\lambda} \in H^{2 \lambda \mid}\left(G_{k, n} ; \mathbf{Q}\right)$ are the Poincare duals of the Schubert cycles (see e.g. [8]). The next result gives a simple relation between the $\sigma_{\lambda}$ and the Schur functions $s_{\lambda}$.

THEOREM 2.4. The isomorphism of 2.3 sends $s_{\lambda}$ to $(-1)^{||_{1}} \sigma_{\lambda}$. 
Proof. From [8, p. 410], we have $c_{i}=(-1)^{i} \sigma_{11 \ldots 1}=(-1)^{i} \sigma_{1^{\prime}}$ in $H^{*}\left(G_{k, n}\right.$; Q). But we have also $\sum_{r=0}^{d}(-1)^{r} \sigma_{1} \sigma_{d-r}=0$ for $d>0$ [8, p. 205]. Thus $\sum_{r=0}^{d} c_{r} \sigma_{d-r}=0$ for $d>0$ : but then $\sigma_{i}=\left(c^{-1}\right)_{i}$ is the image of $(-1)^{i} h_{i}$. Since

$$
\sigma_{\lambda}=\operatorname{det}\left(\sigma_{\lambda_{p}-p+q}\right)_{1 \leqslant p, q \leqslant l(\lambda)}
$$

[8, p. 205], the result follows.

REMARK. The relationship between the $s_{\lambda}$ and the $\sigma_{\lambda}$ was first explained in [12], though the formal similarity between the multiplication rules for the $\sigma_{\lambda}$ (i.e., the classical Schubert calculus) and those for the $s_{\lambda}$ was noted earlier; see [17] for details.

In the sequel we shall write $c^{\lambda}=\operatorname{det}\left(c_{\lambda_{p}-p+q}\right)_{1 \leqslant p, q \leqslant 1}$ and $c_{\lambda}=c_{\lambda_{1}} c_{\lambda_{2}} \cdots c_{\lambda_{1}}$ for a partition $\lambda=\lambda_{1} \lambda_{2} \cdots \lambda_{l}$. By 2.2 and $2.4 c^{\lambda}=(-1)^{|\lambda|} \sigma_{\lambda^{\prime}}$. Thus $H^{2 i}\left(G_{k, n} ; \mathbf{Q}\right)$ has basis $\left\{c^{\lambda} \mid \lambda \in P(i)\right\}$, where $P(i)$ is the set of partitions $\lambda$ with $|\lambda|=i, \lambda_{1} \leqslant k$, and $l(\lambda) \leqslant n$. Now $P(i)$ has a total ordering defined by putting $\mu>\lambda$ if the first nonzero difference $\mu_{r}-\lambda_{r}, 1 \leqslant r \leqslant n$, is positive. It is evident (from expanding the determinant) that

$$
c^{\lambda}=c_{\lambda}+\sum_{\mu \in P(i), \mu>\lambda} D_{\mu} c_{\mu}
$$

for some integers $D_{\mu}$. Thus, we have the following fact.

Proposition 2.5. $H^{2 i}\left(G_{k, n} ; \mathbf{Q}\right)$ has basis $\left\{c_{\lambda} \mid \lambda \in P(i)\right\}$.

3. The hard Lefschetz theorem. In this section we use the hard Lefschetz theorem to establish a result (Theorem 3.3) which "almost" proves Theorem 1.1 by induction. This partial result is the basis for the remainder of the proof.

The hard Lefschetz theorem was first stated by Lefschetz [13] for projective varieties, though the first rigorous proof is due to Hodge [10]. Other references are [18 and 8].

THEOREM 3.1 (HARD LEFSCHETZ THEOREM). Let $M$ be a compact Kähler manifold of complex dimension $d$, with Kähler class $u \in H^{2}(M ; \mathbf{Q})$. Then if $0 \leqslant r \leqslant d$, multiplication by $u^{d-r}$ gives an isomorphism $H^{r}(M ; \mathbf{Q}) \stackrel{\sim}{\rightarrow} H^{2 d-r}(M ; \mathbf{Q})$.

Since $G_{k, n}$ is a Kähler manifold with Kähler class $\sigma_{1}=-c_{1}$, we immediately have the following corollary.

COROllaRy 3.2. Multiplication by $c_{1}^{k n-2 i}, 0 \leqslant 2 i \leqslant k n$, gives an isomorphism

$$
H^{2 i}\left(G_{k, n} ; \mathbf{Q}\right) \stackrel{\sim}{\rightarrow} H^{2 k n-2 i}\left(G_{k, n} ; \mathbf{Q}\right) \text {. }
$$

Thus, $\left\{c_{1}^{k n-2 i} c_{\mu} \mid \mu \in P(i)\right\}$ is a basis for $H^{2 k n-2 i}\left(G_{k, n} ; \mathbf{Q}\right)$.

By Poincare duality, cup product is a nonsingular dual pairing

$$
H^{2 i}\left(G_{k, n} ; \mathbf{Q}\right) \otimes H^{2 k n-2 i}\left(G_{k, n} ; \mathbf{Q}\right) \rightarrow \mathbf{Q} .
$$

Thus, if we define a matrix $M=\left(M_{\mu \lambda}\right)_{\mu, \lambda \in P(i)}$ by $c_{1}^{k n-2 i} c_{\mu} c_{\lambda}=M_{\mu \lambda} c_{k}^{n}$, then 3.2 implies $M$ is nonsingular. This observation is the key to proving the next result. 
THEOREM 3.3. Let $h$ be an endomorphism of $H^{*}\left(G_{k, n} ; \mathbf{Q}\right)$ with $h\left(c_{t}\right)=m^{t} c_{t}$, $1 \leqslant t<i$, for some $m \neq 0$. Then $h\left(c_{i}\right)=m^{i} c_{i}$ or

$$
h\left(c_{i}\right)=-m^{i} c_{i}-2 m^{i} \sum_{\lambda \in P(i), \lambda \neq i} \frac{A_{\lambda i}}{A_{i i}} c_{\lambda},
$$

where the $A_{\lambda i}$ are appropriate (signed) minors of $M$.

Proof. For convenience, let $Q(i)=P(i)-\{i\}$. In view of 2.5 , we can write

$$
h\left(c_{i}\right)=\sum_{\lambda \in P(i)} a_{\lambda} c_{\lambda}
$$

For $\mu \in Q(i)$, applying $h$ to the relation

$$
c_{1}^{k n-2 i} c_{\mu} c_{i}=M_{\mu i} c_{k}^{n}
$$

gives

$$
m^{k n-i} c_{1}^{k n-2 i} c_{\mu}\left(\sum_{\lambda \in P(i)} a_{\lambda} c_{\lambda}\right)=M_{\mu i} m^{k n} c_{k}^{n} .
$$

(Note $c_{k}^{n}$ is a multiple of $c_{1}^{k n}$ by 3.2.) Rewriting everything as a multiple of $c_{k}^{n}$, we get

$$
M_{\mu i}\left(a_{i}-m^{i}\right)+\sum_{\lambda \in Q(i)} M_{\mu \lambda} a_{\lambda}=0 .
$$

Similarly, applying $h$ to $c_{1}^{k n-2 i} c_{i}^{2}=M_{i i} c_{k}^{n}$ yields

$$
M_{i i}\left(a_{i}^{2}-m^{2 i}\right)+2 a^{i} \sum_{\mu \in Q(i)} M_{\mu i} a_{\mu}+\sum_{\mu, \lambda \in Q(i)} M_{\mu \lambda} a_{\mu} a_{\lambda}=0 .
$$

Now multiply (2) by $a_{\mu}$, sum over $\mu \in Q(i)$, and subtract the resulting equation from (3) to get

$$
M_{i i}\left(a_{i}^{2}-m^{2 i}\right)+\left(a_{i}+m^{i}\right) \sum_{\mu \in Q(i)} M_{\mu i} a_{\mu}=0 .
$$

Provided $a_{i} \neq-m^{i}$, we can divide this by $a_{i}+m^{i}$ to obtain

$$
M_{i i}\left(a_{i}-m^{i}\right)+\sum_{\mu \in Q(i)} M_{\mu i} a_{\mu}=0 .
$$

Together with (2) for $\mu \in Q(i)$, this gives a system

$$
\sum_{\lambda \in P(i)} M_{\mu \lambda}\left(a_{\lambda}-\delta_{\lambda i} m^{i}\right)=0, \quad \mu \in P(i),
$$

where $\delta$ denotes Kronecker delta. By the nonsingularity of $M$, we then have $a_{\lambda}=\delta_{\lambda i} m^{i}$, i.e. $h\left(c_{i}\right)=m^{i} c_{i}$.

Now suppose $a_{i}=-m^{i}$. Then (2) gives

$$
\sum_{\lambda \in P(i)} M_{\mu \lambda} a_{\lambda}=m^{i} M_{\mu i}
$$

for $\mu \in Q(i)$. By cofactor expansion,

$$
\sum_{\mu \in P(i)} A_{\nu \mu} M_{\mu \lambda}=\delta_{\nu \lambda} \operatorname{det} M .
$$


Multiply (4) by $A_{i \mu}$ and sum over $\mu \in Q(i)$ to get

$$
\sum_{\mu \in Q(i)} \sum_{\lambda \in P(i)} A_{i \mu} M_{\mu \lambda} a_{\lambda}=m^{i} \sum_{\mu \in Q(i)} A_{i \mu} M_{\mu i} .
$$

By the use of (5), this can be reduced to

$$
A_{i i} \sum_{\lambda \in P(i)} M_{i \lambda} a_{\lambda}=m^{i} A_{i i} M_{i i}-2 m^{i} \operatorname{det} M
$$

Since det $M \neq 0$, evidently $A_{i i} \neq 0$. Equation (6) divided by $A_{i i}$, together with (4) for $\mu \in Q(i)$, gives a system

$$
\sum_{\lambda \in P(i)} M_{\mu \lambda} a_{\lambda}=m^{i} M_{\mu i}-\delta_{i \mu} m^{i} \frac{2 \operatorname{det} M}{A_{i i}}, \quad \mu \in P(i),
$$

which can be solved using (5) to give

$$
a_{\lambda}=m^{i} \delta_{i \lambda}-2 m^{i} \frac{A_{i \lambda}}{A_{i i}} .
$$

In what follows, $h$ will be an endomorphism of $H^{*}\left(G_{k, n} ; \mathbf{Q}\right)$ with $h\left(c_{1}\right)=m c_{1}$, $m \neq 0$. Coefficients $a_{\mu}, \mu \in P(i)$, will be defined by (1) for $1 \leqslant i \leqslant k$. Then Theorem 3.3 has the following consequence.

Corollary 3.4. For $1 \leqslant i \leqslant k, a_{i}= \pm m^{i}$.

Proof. By 3.3, we have $h^{2}\left(c_{1}\right)=m^{2 i} c_{i}$ whenever $h\left(c_{t}\right)=m^{t} c_{t}, t<i$. Iterate to get $h^{N}\left(c_{i}\right)=m^{N i} c_{i}, 1 \leqslant i \leqslant k$, for some $N$. Then the conclusion follows.

We shall set $a_{i}=\varepsilon_{i} m^{i}$ for $1 \leqslant i \leqslant k$, so each $\varepsilon_{i}$ is \pm 1 (note $\varepsilon_{1}=1$ ). By 3.3 , to prove $h\left(c_{1}\right)=m^{i} c_{i}$ it suffices to prove $\varepsilon_{t}=1$ for $t \leqslant i$.

4. Computations in top dimension. By the remarks following 3.4, to establish Theorem 1.1 for $n>k$ it suffices to show $\varepsilon_{i}=1,1 \leqslant i \leqslant k$. In this section we prove $\varepsilon_{2}=1$ for $n>k \geqslant 2$, and compute $h\left(c_{2}\right)$ when $n=k$ and $\varepsilon_{2}=-1$. The method is explicit computation based on a formula (Proposition 4.2) for coefficients in $H^{2 k n}\left(G_{k, n} ; \mathbf{Q}\right)$.

If $\mu$ is a partition without ones, $|\mu| \leqslant k n$, and $\mu_{1} \leqslant k$, we define $M_{\mu}$ by the relation

$$
c_{1}^{k n-|\omega|} c_{\mu}=M_{\mu} c_{k}^{n}
$$

in $H^{2 k n}\left(G_{k, n} ; \mathbf{Q}\right)$. Now suppose $\varepsilon_{2}=-1$. By 3.3,

$$
h\left(c_{2}\right)=-m^{2} c_{2}-2 m^{2} \frac{A_{2.11}}{A_{2.2}} c_{1}^{2}=-m^{2} c_{2}+2 m^{2} \frac{M_{2}}{M_{\varnothing}} c_{1}^{2}
$$

( $\varnothing$ is the empty partition, so $c_{1}^{k n}=M_{\varnothing} c_{k}^{n}$ ). Assume $k n \geqslant 6$ (as when $n>k \geqslant 2$ ). Then applying $h$ to $c_{1}^{k n-6} c_{2}^{3}=M_{222} c_{k}^{n}$, using (1), expanding out the resulting equation and rewriting everything as a multiple of $c_{k}^{n}$, we get

$$
\frac{M_{222}}{M_{2}}-3 \frac{M_{22}}{M_{\varnothing}}+2\left(\frac{M_{2}}{M_{\varnothing}}\right)^{2}=0 .
$$

So $\varepsilon_{2}=-1$ implies (2) for $k n \geqslant 6$ : we shall show (2) cannot hold for $n>k \geqslant 2$. 
First we give a combinatorial description of Poincaré duality in $H^{*}\left(G_{k, n} ; \mathbf{Q}\right)$. Given a partition $\lambda \in P(i)$, the dual partition $\bar{\lambda} \in P(k n-i)$ is defined by $\bar{\lambda}_{q}=k$ $-\lambda_{n+1-q}$. Then the following result holds.

Proposition 4.1. Let $\lambda \in P(i), \mu \in P(k n-i)$. Then in $H^{*}\left(G_{k, n} ; \mathbf{Q}\right), c^{\lambda} c^{\mu}=\delta_{\lambda \mu}^{-} c_{k}^{n}$.

Proof. This follows from the corresponding rule in the Schubert calculus $[8, \mathrm{p}$. 198], using $c^{\lambda}=(-1)^{|\lambda|} \sigma_{\lambda^{\prime}}$.

Now let $\mu$ be a partition. For each box $b$ in the diagram of $\mu$, we define the hook length $H(b)$ by

$$
H(b)=\text { number of boxes below } b+\text { number of boxes to right of } b+1
$$

(so $H(b)$ is the length of the "hook" containing $b$ ). We set

$$
N(\mu)=\frac{|\mu| !}{\prod_{b \in \text { diagram of } \mu} H(b)} .
$$

For example, if $\mu=3321$, the hook lengths of boxes in the diagram of $\mu$ are

\begin{tabular}{|l|l|l|}
\hline 6 & 4 & 2 \\
\hline 5 & 3 & 1 \\
\hline 3 & 1 & \multicolumn{1}{|}{} \\
\cline { 1 - 2 } 1 & \multicolumn{2}{|l}{} \\
\cline { 1 - 2 } & &
\end{tabular}

from which we compute

$$
N(\mu)=\frac{9 !}{2 \cdot 3^{2} \cdot 4 \cdot 5 \cdot 6}=168 .
$$

In fact, $N(\mu)$ is always an integer. The numbers $N(\mu)$ appear in the next result.

Proposition 4.2. Let $\lambda \in P(s)$. Then in $H^{2 k n}\left(G_{k, n} ; \mathbf{Q}\right), c_{1}^{k n-s} c^{\lambda}=N(\bar{\lambda}) c_{k}^{n}$.

Proof. We start with the formula $e_{1}^{r}=\Sigma_{|\mu|=r} N(\mu) s_{\mu}$, which holds in $\Lambda_{k}$ [15, p. 37]. Using the correspondence of $\S 2$ (and the fact $N\left(\mu^{\prime}\right)=N(\mu)$ ), we then have $c_{1}^{r}=\Sigma_{\mu \in P(r)} N(\mu) c^{\mu}$ in $H^{*}\left(G_{k, n} ; \mathbf{Q}\right)$. Now put $r=k n-s$, multiply by $c^{\lambda}$, and use 4.1 to get the conclusion.

In what follows, we put $N_{\mu}=N(\bar{\mu})$. For small $|\mu|$, the ratio $N_{\mu} / N_{\varnothing}$ is fairly easy to compute. For example, we can show (setting $\xi=k n$ and $\eta=n-k$ )

$$
\frac{N_{2}}{N_{\varnothing}}=\frac{\xi-\eta-1}{2(\xi-1)}, \quad \frac{N_{3}}{N_{\varnothing}}=\frac{(\xi-\eta-1)(\xi-2 \eta-4)}{6(\xi-1)(\xi-2)}
$$

and

$$
\frac{N_{22}}{N_{\varnothing}}=\frac{\xi(\xi-\eta-1)(\xi+\eta-1)}{12(\xi-1)(\xi-2)(\xi-3)}
$$


From formulas like these, we obtain expressions for the coefficients $M_{\mu}$. For example, since $c_{2}^{2}=c^{22}+c_{1} c^{3}$ we have $M_{22}=N_{22}+N_{3}$ and thus (noting $M_{\varnothing}=N_{\varnothing}$ )

$$
\begin{aligned}
\frac{M_{22}}{M_{\varnothing}} & =\frac{\xi(\xi-\eta-1)(\xi+\eta-1)}{12(\xi-1)(\xi-2)(\xi-3)}+\frac{(\xi-\eta-1)(\xi-2 \eta-4)}{6(\xi-1)(\xi-2)} \\
& =\frac{(\xi-\eta-1)\left[\xi^{2}-5 \xi+8-\eta(\xi-4)\right]}{4(\xi-1)(\xi-2)(\xi-3)} .
\end{aligned}
$$

With these techniques, a lengthy but straightforward computation shows

$$
\frac{M_{222}}{M_{2}}-3 \frac{M_{22}}{M_{\varnothing}}+2\left(\frac{M_{2}}{M_{\varnothing}}\right)^{2}=\frac{2 \eta(\xi+5)(\xi+\eta-1)}{(\xi-1)^{2}(\xi-2)(\xi-3)(\xi-4)(\xi-5)} .
$$

If $n>k \geqslant 2$ (hence $\xi \geqslant 6$ and $\eta>0$ ), the right-hand side is clearly positive. This contradicts (2), so $\varepsilon_{2} \neq-1$ for $n>k \geqslant 2$. If $n=k$,

$$
\frac{M_{2}}{M_{\varnothing}}=\frac{N_{2}}{N_{\varnothing}}=\frac{\xi-1}{2(\xi-1)}=\frac{1}{2}
$$

and (1) is $h\left(c_{2}\right)=-m^{2} c_{2}+m^{2} c_{1}^{2}$. We have established the following result.

THEOREM 4.3. If $k \geqslant 2$, then $\varepsilon_{2}=1$ when $n>k$. If $n=k$ and $\varepsilon_{2}=-1$, then $h\left(c_{2}\right)=m^{2}\left(-c_{2}+c_{1}^{2}\right)$.

Since Theorem 1.1 is trivial for $k=1$ (the case of projective space), we immediately have the following corollary.

COROLlary 4.4. Theorem 1.1 holds if $k \leqslant 2$.

5. Comparison of coefficients. In this section we obtain information on the $\varepsilon_{i}$, show some of the coefficients $a_{\mu}$ to be zero, and establish Theorem 1.1 in some special cases by examining the images under $h$ of the relations

$$
R_{i}=\left(c^{-1}\right)_{n+i}, \quad 1 \leqslant i \leqslant k,
$$

in $\mathbf{Q}\left[c_{1}, \ldots, c_{k}\right]$. Most of the arguments of this section and the following one are adapted from [3].

If $h$ is a well-defined endomorphism of $H^{*}\left(G_{k, n} ; \mathbf{Q}\right)$, we must have relations

$$
h\left(R_{i}\right)=K_{i} R_{i}+\sum_{1 \leqslant|\mu|<i} K_{\mu}^{(i)} c_{\mu} R_{i-|\mu|}
$$

in $\mathbf{Q}\left[c_{1}, \ldots, c_{k}\right]$ for $1 \leqslant i \leqslant k$. On the other hand, we can write $R_{i}$ explicitly in terms of the $c_{\lambda}$ and then use

$$
h\left(c_{t}\right)=\sum_{\mu \in P(t)} a_{\mu} c_{\mu}, \quad 1 \leqslant t \leqslant k
$$

to write $h\left(R_{i}\right)$. It will be convenient to introduce some additional notation.

For any partition $\lambda$, set $m_{i}(\lambda)=$ order of $\left\{q \mid \lambda_{q}=i\right\}$. Then $m_{1}(\lambda), m_{2}(\lambda), \ldots$ uniquely determine $\lambda$, and $m_{1}(\lambda)+m_{2}(\lambda)+\cdots=l(\lambda)$. We set

$$
C(\lambda)=(-1)^{l(\lambda)} \frac{l(\lambda) !}{m_{1}(\lambda) ! m_{2}(\lambda) ! \cdots m_{k}(\lambda) !}
$$


for partitions $\lambda$ with $\lambda_{1} \leqslant k$. Then a formal computation using the multinomial theorem shows

$$
R_{i}=\left(c^{-1}\right)_{n+i}=\sum_{|\lambda|=n+i, \lambda_{1} \leqslant k} C(\lambda) c_{\lambda}
$$

Thus

(2) $\quad h\left(R_{i}\right)=\sum_{|\lambda|=n+i, \lambda_{1} \leqslant k} C(\lambda) h\left(c_{\lambda}\right)=\sum_{|\lambda|=n+i, \lambda_{1} \leqslant k} C(\lambda) \prod_{t=1}^{l(\lambda)}\left(\sum_{\mu \in P\left(\lambda_{t}\right)} a_{\mu} c_{\mu}\right)$.

By comparing coefficients of specific terms in (1) and (2), we shall obtain relations in the $a_{\mu}$.

In what follows, we define $p$ and $r$ by $n=p k+r, 0 \leqslant r<k$, and set $\alpha=\varepsilon_{k-1} \varepsilon_{k}$.

Proposition 5.1. If $i \geqslant \min \{r+1, k-r+1\}$, then $\varepsilon_{i}=\alpha^{k-i} \varepsilon_{k}$.

Proof. First we examine the coefficient of $c_{r+u+1} c_{k v-u} c_{k}^{p-1}$ in $h\left(R_{i}\right)$ for $0 \leqslant u \leqslant$ $k-r+1$. From (1) the coefficient is

$$
C\left(k^{p-1} k-u r+u+1\right) K_{1},
$$

(recall that $k^{p-1}$ means $k$ repeated $p-1$ times) while from (2) it is

$$
C\left(k^{p-1} k-u r+u+1\right) a_{r+u+1} a_{k-u} a_{k}^{p-1} .
$$

Thus $K_{1}=a_{r+u+1} a_{k-u} a_{k}^{p-1}$ for $0 \leqslant u \leqslant k-r-1$. It follows that

$$
\varepsilon_{r+1} \varepsilon_{k}=\varepsilon_{r+2} \varepsilon_{k-1}=\cdots=\varepsilon_{k} \varepsilon_{r+1} .
$$

A similar analysis of the coefficient of $c_{r+u+2} c_{k-u} c_{k}^{p-1}$ in $h\left(R_{2}\right), 0 \leqslant u \leqslant k-r-2$, shows $K_{2}=a_{r+u+2} a_{k-u} a_{k}^{p-1}$ and thus

$$
\varepsilon_{r+2} \varepsilon_{k}=\varepsilon_{r+3} \varepsilon_{k-1}=\cdots=\varepsilon_{k} \varepsilon_{r+2} .
$$

Now work back and forth between (3) and (4) to obtain $\varepsilon_{i}=\alpha^{k-i} \varepsilon_{k}$ for $i \geqslant r+1$.

To finish the proof, we need only consider the case $k<2 r$. Here we examine the coefficient of $c_{k-r+u+1} c_{r} c_{k-u} c_{k}^{p-1}$ in $h\left(R_{k-r+1}\right), 0 \leqslant u \leqslant r-1$, to find

$$
a_{k-r+u+1} a_{r} a_{k-u} a_{k}^{p-1}=K_{k-r+1} .
$$

Examining the coefficient of $c_{k-r+u+1} c_{r} c_{k-u} c_{k}^{p-1}$ in $h\left(R_{k-r+2}\right), 0 \leqslant u \leqslant r-2$, gives

$$
a_{k-r+u+2} a_{r} a_{k-u} a_{k}^{p-1}= \begin{cases}K_{k-r+2}, & 2 r>k+1, \\ K_{k-r+2}-\frac{1}{p+2} K_{r}^{(k-r+2)}, & 2 r=k+1 .\end{cases}
$$

From these equations we get ones corresponding to (3) to (4), and we proceed as above to show $\varepsilon_{i}=\alpha^{k-i} \varepsilon_{k}$ for $i \geqslant k-r+1$.

In the case $r=0,5.1$ is enough to prove Theorem 1.1.

COROLlaRY 5.2. If $n=p k$, then Theorem 1.1 holds.

Proof. By 5.1, $\varepsilon_{i}=\alpha^{k-i} \varepsilon_{k}$ for $1 \leqslant i \leqslant k$. Since $\varepsilon_{i}=1,1=\alpha^{k-1} \varepsilon_{k}$ and so $\varepsilon_{i}=\alpha^{i+1}$. Thus $\varepsilon_{2}=\alpha$. If $\alpha=\varepsilon_{2}=1$, then $\varepsilon_{i}=1$ for $1 \leqslant i \leqslant k$ and $h\left(c_{i}\right)=m^{i} c_{i}, 1 \leqslant i \leqslant k$, by the remarks following 3.4 . 
Now suppose $\varepsilon_{2}=-1$. Then $n=k$ and

$$
h\left(c_{2}\right)=m^{2}\left(-c_{2}+c_{1}^{2}\right)=m^{2}\left(c^{-1}\right)_{2}
$$

by 4.3. Let $\Theta: H^{*}\left(G_{k, k} ; \mathbf{Q}\right) \rightarrow H^{*}\left(G_{k, k} ; \mathbf{Q}\right)$ be defined by

$$
\Theta\left(c^{\mu}\right)=(-1)^{|\mu|} c^{\mu^{\prime}}=\sigma_{\mu} .
$$

Then $\Theta$ is an involution taking $c_{i}$ to $\left(c^{-1}\right)_{i}$. We have $\Theta h\left(c_{1}\right)=m\left(c^{-1}\right)_{1}=-m c_{1}$ and $\Theta h\left(c_{2}\right)=m^{2} c_{2}=(-m)^{2} c_{2}$. But then (if we replace $m$ by $-m$ ) $\Theta h$ is an endomorphism with $\varepsilon_{1}=\varepsilon_{2}=1$, so

$$
\Theta h\left(c_{i}\right)=(-m)^{i} c_{i}, \quad 1 \leqslant i \leqslant k,
$$

by the argument of the preceding paragraph. Hence $h\left(c_{i}\right)=(-m)^{i}\left(c^{-1}\right)_{i}$ for $1 \leqslant i \leqslant k$, and Theorem 1.1 holds.

Henceforth we assume $n>k$; by 4.4 , we can assume $k \geqslant 3$. Let $f=[k / 2]$. In the remainder of this section we prove $a_{k k-f}=0, \varepsilon_{f} \varepsilon_{k-f}=\varepsilon_{k}$, and $\varepsilon_{k}=\alpha^{k}$. The arguments depend on the parity of $k$ : we consider first the case where $k$ is even.

Proposition 5.3. If $k=2 f$, then $\varepsilon_{k}=1$ and $a_{f f}=0$.

Proof. By the preceding result, we can assume $r \neq 0$. In this case,

$$
2 a_{f f}=(p+2)\left(a_{f}^{2}-a_{k}\right) \text {. }
$$

This can be established by comparing coefficients of $c_{f}^{2} c_{f+1} c_{k}^{p-1}$ and $c_{f+1} c_{k}^{p}$ in $h\left(R_{f-r+1}\right)$ if $0<r \leqslant f$, or by comparing coefficients of $c_{f}^{2} c_{k}^{p}$ and $c_{k}^{p+1}$ in $h\left(R_{k-r}\right)$ if $f \leqslant r<k$. Thus $\varepsilon_{k}=1$ if and only if $a_{f f}=0$. The remainder of the proof divides into three cases.

Case 1. $0<r<f$. Compare coefficients of $c_{f}^{3} c_{k}^{p-1}$ and $c_{f} c_{k}^{p}$ in $h\left(R_{f-r}\right)$ to get $6 a_{f f}=(p+2)\left(a_{f}^{2}-a_{k}\right)$, and compare this with (5) to obtain the conclusion.

Case 2. $r=f$. We suppose $f \geqslant 3$ : the case $r=f=2$ requires a special argument, which is omitted. Let $1 \leqslant t \leqslant f-1$. Compare coefficients of $c_{t} c_{f} c_{k}^{p}$ and $c_{t+f} c_{k}^{p}$ in $h\left(R_{t}\right)$ to get

$$
a_{f t}=(p+2)\left(a_{t} a_{f}-a_{t+f}\right) .
$$

Now compare coefficients of $c_{t} c_{f}^{3} c_{k}^{p-1}$ and $c_{t+f} c_{k}^{p}$ to get

$$
6 a_{f t} a_{f f}-6(p+2) a_{t} a_{f} a_{f f}-3(p+2) a_{f}^{2} a_{f t}=(p+2)(p+3)\left(a_{t+f} a_{k}-a_{t} a_{f}^{3}\right) .
$$

Use (5) and (6) to substitute for $a_{f f}$ and $a_{f t}$ respectively. The result is

$$
\varepsilon_{t+f}=\varepsilon_{t} \varepsilon_{f} \varepsilon_{k}, \quad 1 \leqslant t \leqslant f-1 .
$$

If $\varepsilon_{k}=-1$, then (7) together with $\varepsilon_{2}=1$ gives

$$
\varepsilon_{f+2}=-\varepsilon_{f} \text {. }
$$

(This uses $f \geqslant 3$.) Compare coefficients of $c_{f+1}^{3} c_{k}^{p-1}$ and $c_{f} c_{f+1} c_{f+2} c_{k}^{p-1}$ in $h\left(R_{3}\right)$ (again using $f \geqslant 3$ ) to get $a_{f+1}^{2}=a_{f} a_{f+2}$ : but then $\varepsilon_{f} \varepsilon_{f+2}=1$, contradicting (8). So $\varepsilon_{k}=1$.

Case 3. $f<r<k$. Compare coefficients of $c_{f} c_{f+1} c_{k}^{p}$ and $c_{f}^{3} c_{f+1} c_{k}^{p-1}$ in $h\left(R_{k-r+1}\right)$ to get $6 a_{f f}=(p+3)\left(a_{f}^{2}-a_{k}\right)$. Together with (5), this gives the conclusion. 
We note that the argument of Case 2 above can be improved to yield the following result.

Proposition 5.4. If $k=2 f$ and $r=f$, then Theorem 1.1 holds.

Proof. As in Case 2, we assume $f \geqslant 3$ and omit the special argument for $r=f=2$. Now $\varepsilon_{k}=1$ by 5.3 , so (7) is

$$
\varepsilon_{t+f}=\varepsilon_{t} \varepsilon_{f}, \quad 1 \leqslant t \leqslant f-1 .
$$

By 5.1 ,

$$
\varepsilon_{t+f}=\alpha^{k-f-t} \varepsilon_{k}=\alpha^{f+t}
$$

for $1 \leqslant t \leqslant f$. Thus $\varepsilon_{t} \varepsilon_{f}=\alpha^{f+t}, 1 \leqslant t \leqslant f-1$. Since $\varepsilon_{1}=\varepsilon_{2}=1$, this means $\alpha^{f+1}=$ $\varepsilon_{f}=\alpha^{f+2}$, i.e. $\alpha=1$. But then $\varepsilon_{f}=1$, and by (10) $\varepsilon_{f+t}=1$ for $1 \leqslant t \leqslant f$. Hence (9) becomes $\varepsilon_{t}=1,1 \leqslant t \leqslant f-1$, and we have $\varepsilon_{i}=1$ for $1 \leqslant i \leqslant k$. Theorem 1.1 then follows in this case.

Now we turn to the case where $k$ is odd.

Proposition 5.5. If $k=2 f+1$, then $\varepsilon_{k}=\alpha$.

Proof. We can assume $r \neq 0$. If $1 \leqslant r \leqslant f+1$, we compare coefficients of $c_{f+1}^{3} c_{k}^{p-1}$ in $c_{f+2} c_{k}^{p}$ in $h\left(R_{f-r+2}\right)$ to get

$$
a_{f+1}^{3} a_{k}^{p-1}=a_{f+2} a_{k}^{p} .
$$

If $f+2 \leqslant r \leqslant k-1$, we compare coefficients of $c_{f+1}^{4} c_{k}^{p-1}$ and $c_{f+1} c_{f+2} c_{k}^{p}$ in $h\left(R_{k-r+2}\right)$ to get

$$
a_{f+1}^{4} a_{k}^{p-1}=a_{f+1} a_{f+2} a_{k}^{p} .
$$

Either equation implies $\varepsilon_{k}=\varepsilon_{f+1} \varepsilon_{f+2}$, and $\varepsilon_{f+1} \varepsilon_{f+2}=\alpha$ by 5.1.

Proposition 5.6. If $k=2 f+1$, then $\varepsilon_{f} \varepsilon_{f+1}=\alpha$ and $a_{f+1 f}=0$.

Proof. Unless $r=f$ or $r=f+1, \varepsilon_{f} \varepsilon_{f+1}=\alpha$ by 5.1. We consider two cases.

Case $1.0<r \leqslant f$. Compare coefficients of $c_{f+1} c_{k}^{p}$ and $c_{f} c_{f+1}^{2} c_{k}^{p-1}$ in $h\left(R_{f-r+1}\right)$ to get

$$
2 a_{f+1 f}=(p+2)\left(a_{f} a_{f+1}-a_{k}\right) .
$$

Except for $r=f$, we have $\varepsilon_{f} \varepsilon_{f+1}=\alpha=\varepsilon_{k}$ and the conclusion follows. Suppose now $r=f \geqslant 2(r=f=1$ requires a special argument). Then compare coefficients of $c_{f+2} c_{k}^{p}$ and $c_{f} c_{f+1} c_{f+2} c_{k}^{p-1}$ in $h\left(R_{2}\right)$ to obtain

$$
a_{f+1 f}=(p+2)\left(a_{f} a_{f+1}-a_{k}\right) .
$$

Together with (11), this gives the conclusion.

Case 2. $f+1 \leqslant r<k$. Comparing coefficients of $c_{k}^{p+1}$ and $c_{f+1} c_{k}^{p}$ in $h\left(R_{k-r}\right)$ yields

$$
a_{f+1 f}=(p+2)\left(a_{f} a_{f+1}-a_{k}\right)
$$


which proves the conclusion unless $r=f+1$. Suppose $r=f+1, f \geqslant 2$ (again $f=1$ requires a special argument). Then compare coefficients of $c_{k-1} c_{k}^{p}$ and $c_{f}^{2} c_{k}^{p}$ in $h\left(R_{f-1}\right)$ to get

$$
2 a_{f f}=(p+2)\left(a_{f}^{2}-a_{k-1}\right) .
$$

Now $\varepsilon_{k-1}=1$ by 5.5 , so $a_{f f}=0$. Using this fact, analyze the coefficient of $c_{f}^{3} c_{f+1} c_{k}^{p-1}$ to obtain $3 a_{f+1 f}=(p+3)\left(a_{f} a_{f+1}-a_{k}\right)$. Along with (12), this implies the conclusion.

We close this section with the following improvement of 5.1 .

THEOREM 5.7. If $s \geqslant \min \{k-r+1, r+1, f\}$, then $\varepsilon_{s}=\alpha^{s}$.

Proof. Suppose first that $s \geqslant \min \{k-r+1, r+1\}$. By 5.1, $\varepsilon_{s}=\alpha^{k-s} \varepsilon_{k}$. If $k$ is even, $\varepsilon_{k}=1$ by 5.3 and $\alpha^{k-s}=\alpha^{s}$, so the conclusion follows. If $k$ is odd, $\varepsilon_{k}=\alpha$ by 5.6 and $\alpha^{k-s}=\alpha^{s+1}$, so $\varepsilon_{s}=\alpha^{s+1} \alpha=\alpha^{s}$.

Now $\min \{k-r+1, r+1\}>f$ only if $r=f$, or if $r=k+1$ and $k$ is odd; we finish the proof by checking these cases. If $r=f$ and $k$ is even, use 5.4. If $k$ is odd and $r=f$ or $r=f+1,5.6$ gives $\varepsilon_{f} \varepsilon_{f+1}=\alpha$, from which the conclusion follows.

6. Completion of the proof. In this section we complete the proof of Theorem 1.1, using the comparison-of-coefficients technique. We first establish relations among some of the coefficients $a_{\mu}$ with $l(\mu)=2$, and then use them to show $\varepsilon_{i}=1$, $1 \leqslant i \leqslant k$, when $n>k$.

Proposition 6.1. If $\min \{k-r+1, r+1\}<v \leqslant u<k$ and $u+v>k+1$, then

$$
a_{v k-u} a_{u}+a_{u k-u} a_{v}=a_{v-1 k-u} a_{u+1} .
$$

Proof. We consider four cases.

Case 1. $v \geqslant r+1$. Comparing coefficients of $c_{k-u} c_{v} c_{u} c_{k}^{p-1}$ and $c_{k-u} c_{v-1} c_{u+1} c_{k}^{p-1}$ in $h\left(R_{v-r}\right)$ yields

$$
\begin{aligned}
-a_{v k-u} a_{u}-a_{u k-u} a_{v}+(p+2) a_{k-u} a_{v} a_{u} \\
=-a_{v-1 k-u} a_{u+1}+(p+2) a_{k-u} a_{v-1} a_{u+1} .
\end{aligned}
$$

Because of the restrictions on $u$ and $v$, we can apply 5.7 to get $a_{v-1} a_{u+1}=$ $\alpha^{u+v} m^{u+v}=a_{v} a_{u}$ and thus (1) reduces to the conclusion.

It remains to consider the cases where $v \leqslant r$. In view of the hypothesis, $v \leqslant r$ implies $\min \{k-r+1, r+1\}=k-r+1$, and thus $k-r+1<v \leqslant r$. In all the remaining cases, the argument is based on comparison of the coefficients of $c_{k-u} c_{v} c_{u} c_{r-1} c_{k}^{p-1}$ and $c_{k-u} c_{v-1} c_{u+1} c_{r-1} c_{k}^{p-1}$ in $h\left(R_{v-1}\right)$.

Case 2. $v=k-r+2 \leqslant r$. Here $u+v>k+1$ implies $u>r-1$. Comparison of the coefficients indicated above yields

$$
\begin{aligned}
& -a_{v k-u} a_{r-1} a_{u}-a_{u k-u} a_{v} a_{r-1}+(p+3) a_{k-u} a_{v} a_{r-1} a_{u} \\
& \quad=-a_{v-1 k-u} a_{r-1} a_{u+1}-a_{r-1 v-1} a_{k-u} a_{u+1}+(p+3) a_{k-u} a_{v-1} a_{r-1} a_{u+1},
\end{aligned}
$$

from which follows

$$
\left(a_{v k-u} a_{u}+a_{u k-u} a_{v}\right) a_{r-1}=a_{v-1 k-u} a_{u+1} a_{r-1}+a_{r-1 v-1} a_{k-u} a_{u+1} .
$$


Thus, to get the conclusion we must show $a_{r-1 v-1}=a_{r-1 k-r+1}$ is zero. Compare coefficients of $c_{k}^{p+1}$ and $c_{k-r+1} c_{r-1} c_{k}^{p}$ in $h\left(R_{k-r}\right)$ to obtain

$$
a_{r-1 k-r+1}=(p+2)\left(a_{k}-a_{r-1} a_{k-r+1}\right) \text {, }
$$

and note the right-hand side is zero by 5.7 .

Case 3. $k-r+2<v \leqslant u<r-1$. Compare the indicated coefficients to get

$$
\begin{aligned}
& -a_{v k-u} a_{u} a_{r-1}-a_{u k-u} a_{v} a_{r-1}+(p+3) a_{k-u} a_{v} a_{u} a_{r-1} \\
& =-a_{v-1 k-u} a_{u+1} a_{r-1}+(p+3) a_{k-u} a_{v-1} a_{u+1} a_{r-1},
\end{aligned}
$$

from which the conclusion follows as in Case 1 .

Case 4. $k-r+2<v \leqslant r, u \geqslant r-1$. Comparison of the indicated coefficients yields

$$
\begin{aligned}
& -a_{v k-u} a_{r-1} a_{u}-a_{u k-u} a_{v} a_{r-1}-a_{r-1 k-u} a_{v} a_{u}+(p+3) a_{k-u} a_{v} a_{r-1} a_{u} \\
& \quad=-a_{v-1 k-u} a_{r-1} a_{u+1}-a_{r-1 k-u} a_{v-1} a_{u+1}+(p+3) a_{k-u} a_{v-1} a_{r-1} a_{u+1} .
\end{aligned}
$$

from which the conclusion follows as above.

Proposition 6.2. If $f+1<u<k$, then $a_{f+1 k-u} a_{u}+a_{u k-u} a_{f+1}=a_{f k-u} a_{u+1}$.

Proof. This follows from 6.1 unless $\min \{k-r+1, r+1\}>f$, which happens only if $r=f$ or if $r=f+1$ and $k$ is odd. The case $r=f, k$ even, was disposed of in 5.4. If $r=f$ and $k$ is odd, the argument of Case 1 above applies (with $v=f+1$ ). Finally, suppose $r=f+1$ and $k$ is odd. Then comparing coefficients of $c_{k-u} c_{f} c_{f+1} c_{u} c_{k}^{p-1}$ and $c_{k-u} c_{f}^{2} c_{u+1} c_{k}^{p-1}$ in $h\left(R_{f}\right)$, using $a_{f+1 f}=a_{f f}=0$ (see 5.6, Case 2), gives

$$
\begin{array}{r}
-a_{u k-u} a_{f} a_{f+1}-a_{f k-u} a_{f+1} a_{u}-a_{f+1 k-u} a_{f} a_{u}+(p+3) a_{k-u} a_{f} a_{f+1} a_{u} \\
=-2 a_{f k-u} a_{f} a_{u+1}+(p+3) a_{k-u} a_{f}^{2} a_{u+1} .
\end{array}
$$

Using 5.7, we then obtain the conclusion as in the proof of 6.1 .

We use Propositions 6.1 and 6.2 to deduce the following result.

THEOREM 6.3. If $\min \{k-r+1, r+1, f\} \leqslant w \leqslant u<k$ and $w+u>k$, then

$$
\alpha^{u-w} m^{u-w} a_{w k-u}=(u-w+1) a_{u k-u} .
$$

Proof. We proceed by induction on $u-w$. For $u=w$ the conclusion is trivial. Suppose the result holds for $u-w<i$, and take $u, w$ with $u-w=i$ and which satisfy the hypothesis. Then by 6.1 with $v=w+1$ (or by 6.2 if $w=f$ ),

$$
a_{w k-u} a_{u+1}=a_{w+1 k-u} a_{u}+a_{u k-u} a_{w+1} .
$$

Divide by $a_{w+1}$, using 5.7 , to get

$$
\alpha^{u-w} m^{u-w} a_{w k-u}=\alpha^{u-w+1} m^{u-w+1} a_{w+1 k-u}+a_{u k-u} .
$$

By the induction hypothesis,

$$
\alpha^{u-w+1} m^{u-w+1} a_{w+1 k-u}=(u-w) a_{u k-u} .
$$

Substitute this in (2) to obtain the conclusion.

Now we complete the proof of Theorem 1.1. 
LEMMA 6.4. For $i \geqslant 2, \varepsilon_{i}=\alpha^{i}$.

Proof. We can assume $\min \{k-r, r, f-1\} \geqslant 2$, since otherwise the result follows from 5.7. Let $2 \leqslant i \leqslant \min \{k-r, r, f-1\}$, and compare coefficients of $c_{i} c_{k-i} c_{k}^{p}$ and $c_{i} c_{k-i+1} c_{k-1} c_{k}^{p-1}$ in $h\left(R_{k-r}\right)$ to get

$$
a_{k-i i} a_{k}=(p+2)\left(a_{k-i} a_{k}-a_{k-i+1} a_{k-1}\right) a_{i} \text {. }
$$

The right-hand side is zero by 5.7 , so $a_{k-i i}=0$. Then 6.3 implies $a_{r+1 i}=0$, so comparing coefficients of $c_{r+1} c_{k}^{p}$ and $c_{i} c_{r+1} c_{k-1} c_{k-1} c_{k}^{p}$ in $h\left(R_{i}\right)$ yields $a_{r+i} a_{k}=$ $a_{i} a_{r+1} a_{k-1}$. Apply 5.7 to this to get $\varepsilon_{i}=\alpha^{i}$ for $2 \leqslant i \leqslant \min \{k-r, r, f-1\}$. Then the conclusion follows (again using 5.7).

THEOREM 6.5. If $k<n$, then $h\left(c_{i}\right)=m^{i} c_{i}, 1 \leqslant i \leqslant k$.

Proof. We must prove $\varepsilon_{i}=1$ for $1 \leqslant i \leqslant k$ : in view of the preceding lemma, it suffices to show $\alpha=1$. By 5.2, we can assume $r \neq 0$. The case $r=f, k$ even, was disposed of in 5.4. This leaves three cases.

Case 1. $0<r<f, k$ even. Examine the coefficients of $c_{f+1} c_{k}^{p}$ and $c_{1} c_{f} c_{k}^{p}$ in $h\left(R_{f-r+1}\right)$ to obtain $a_{f+1} a_{k}^{p}=K_{f-r+1}$ and $(p+2)\left(a_{f+1}-a_{1} a_{f}\right) a_{k}^{p}+a_{f 1} a_{k}^{p}=$ $K_{1}^{(f-r+1)}$. Using these facts, together with $a_{f f}=0$ (by 5.3), analyze the coefficient of $c_{1} c_{f}^{3} c_{k}^{p-1}$ to get

$$
2 a_{f 1}=a_{1} a_{f}-a_{f+1} .
$$

Now compare coefficients of $c_{f+1} c_{f+r} c_{k}^{p-1}$ and $c_{1} c_{f} c_{f+r} c_{k}^{p-1}$ in $h\left(R_{1}\right)$ to get

$$
a_{f 1} a_{f+r}+a_{f+r 1} a_{f}=(p+2)\left(a_{1} a_{f}-a_{f+1}\right) a_{f+r} .
$$

We can use (3) to reduce this to

$$
a_{f+r 1} a_{f}=(2 p+3) a_{f 1} a_{f+r} .
$$

Now use 6.3 to rewrite $a_{f+r 1}$ and $a_{f 1}$ in terms of $a_{k-11}$, and substitute into (4) to obtain $(f-r) a_{k-11}=(2 p+3) f a_{k-11}$ and thus $a_{k-11}=0$. Then $a_{f 1}=0$, and since $2 a_{f 1}=a_{1} a_{f}-a_{f+1}=(1-\alpha) \alpha^{f} m^{f+1}$ by (3), we have $\alpha=1$.

Case 2. $0<r \leqslant f, k$ odd. Examine the coefficients of $c_{f+2} c_{k}^{p}$ and $c_{1} c_{f+1} c_{k}^{p}$ in $h\left(R_{f-r+2}\right)$ to get $a_{f+2} a_{k}^{p}=K_{f-r+2}$ and

$$
(p+2)\left(a_{f+2}-a_{1} a_{f+1}\right) a_{k}^{p}+a_{f+11} a_{k}^{p}=K_{1}^{(f-r+2)} .
$$

(if $r=1$, it is also necessary to examine the coefficient of $c_{f} c_{f+1} c_{f+2} c_{k}^{p-1}$ to show $K_{f}^{(f+1)}=0$ ). Then analyze the coefficient of $c_{1} c_{f} c_{f+1}^{2} c_{k}^{p-1}$, using $a_{f+1 f}=0$ (by 5.6), to get

$$
a_{f 1} a_{f+1}+a_{f+11} a_{f}=\left(a_{1} a_{f+1}-a_{f+2}\right) a_{f} .
$$

Now use 6.3 to rewrite $a_{f+11}$ and $a_{f 1}$ in terms of $a_{k-11}$ : substitute in the above equation to get

$$
k a_{k-11}=\left(a_{1} a_{f+1}-a_{f+2}\right) \alpha^{f-1} m^{f-1} .
$$

Compare coefficients of $c_{1} c_{f+1} c_{f+r} c_{k}^{p-1}$ and $c_{f+2} c_{f+r} c_{k}^{p-1}$ in $h\left(R_{1}\right)$ to obtain

$$
\left(1+\delta_{1 r}\right) a_{f+11}=(p+2)\left(a_{1} a_{f+1}-a_{f+2}\right),
$$


where $\delta$ denotes Kronecker delta. Multiply by $\alpha^{f-1} m^{f-1}$ and use 6.3 to get

$$
\left(1+\delta_{1 r}\right) a_{k-11}=(p+2)\left(a_{1} a_{f+1}-a_{f+2}\right) \alpha^{f-1} m^{f-1} \text {. }
$$

Comparison with (5) gives $\left(1+\delta_{1 r}\right) a_{k-11}=(p+2) k a_{k-11}$. Since $p \geqslant 1$, this implies $a_{k-11}=0$. But then by (5),

$$
a_{1} a_{f+1}-a_{f+2}=(1-\alpha) \alpha^{f+1} m^{f+2}=0,
$$

so $\alpha=1$.

Case 3. $f<r<k$. Compare the coefficients of $c_{1} c_{r} c_{k}^{p}$ and $c_{1} c_{f} c_{k-f} c_{r} c_{k}^{p-1}$ in $h\left(R_{1}\right)$, using $a_{k-f f}=0$ and $\varepsilon_{f} \varepsilon_{k-f}=\varepsilon_{k}$ (by 5.3 or 5.6), to get

$$
a_{r 1} a_{k}=(p+2)\left(a_{f 1} a_{k-f}+a_{k-f 1} a_{f}\right) a_{r} .
$$

We can use 6.3 to write $a_{r 1}, a_{f 1}$, and $a_{k-f 1}$ in terms of $a_{k-11}$ : substitute into the above equation to get $(k-r) a_{k-11}=(p+2) k a_{k-11}$ and thus $a_{k-11}=0$. Hence $a_{r 1}=0$, and comparison of coefficients of $c_{r+1} c_{k}^{p}$ and $c_{1} c_{r} c_{k}^{p}$ in $h\left(R_{1}\right)$ using this fact yields $a_{r+1}=a_{1} a_{r}$. Then $\varepsilon_{r+1}=\varepsilon_{r}$ : by 6.4 , this means $\alpha=1$.

7. Applications. Theorem 1.1 classifies all automorphisms of $H^{*}\left(G_{k, n} ; \mathbf{Q}\right)$. Since $H^{*}\left(G_{k, n} ; \mathbf{Z}\right)$ has no torsion, Theorem 1.1 remains true if $\mathbf{Q}$ is replaced by $\mathbf{Z}$. Either version establishes homotopy rigidity (in the sense of Liulevicius) for all complex Grassmann manifolds, by the main results of [14 and 1] respectively.

Let $\left(G_{k, n}\right)_{0}$ denote the rationalization of $G_{k, n}$ (for definition see [9]). Then the first theorem of [6] implies the following result.

THEOREM 7.1. The monoid of homotopy classes $\left[\left(G_{k, n}\right)_{0},\left(G_{k, n}\right)_{0}\right]$ is anti-isomorphic to the monoid of endomorphisms of $H^{*}\left(G_{k, n} ; \mathbf{Q}\right)$ via the canonical map.

Thus, Theorem 1.1 can be interpreted as a classification of homotopy classes of self-homotopy equivalences of $\left(G_{k, n}\right)_{0}$. Using results in [7], it then follows that $G_{k, n}$ is generically rigid (i.e., any space in the genus of $G_{k, n}$ is homotopy equivalent to $G_{k, n} ;$ see [7] for definitions).

Conjecture 1.2, if true, would completely classify the endomorphisms of $H^{*}\left(G_{k, n} ; \mathbf{Q}\right)$. By 7.1 , this would give a homotopy classification of self-maps of $\left(G_{k, n}\right)_{0}$. We see another consequence in the next result.

Proposition 7.2. Suppose Conjecture 1.2 holds for $G_{k, n}$. If $k<n$ and $k n$ is even, then $G_{k, n}$ has the fixed-point property.

Proof. Let $f$ be a self-map of $G_{k, n}$. Assuming Conjecture 1.2,

$$
f^{*}\left(c_{i}\right)=m^{i} c_{i}, \quad 1 \leqslant i \leqslant k,
$$

for some $m$. A straightforward computation (see [5]) shows that the Lefschetz number of $f$ is nonzero for any $m$. Thus $f$ has a fixed point by the Lefschetz fixed-point theorem.

Remarks. (1) As noted in $\S 1$, Conjecture 1.2 is known to hold if $k \leqslant 3$ or $n \geqslant 2 k^{2}-k-1$.

(2) If $k=n$ or $k n$ is odd, then $G_{k, n}$ admits fixed-point-free maps. 


\section{REFERENCES}

1. A. Back, Homotopy rigidity for Grassmannians, Proc. Amer. Math. Soc. 80 (1980), 327-332.

2. A. Borel, Sur la cohomologie des espaces fibrés principaux et des espaces homogènes de groupes de Lie compacts, Ann. of Math. (2) 57 (1953), 115-207.

3. S. Brewster, Automorphisms of the cohomology ring of finite Grassmann manifolds, Ph.D. Thesis, Ohio State University, 1978.

4. S. Brewster and W. Homer, Rational automorphisms of Grassmann manifolds, Proc. Amer. Math. Soc. 88 (1983), 181-183.

5. H. Glover and W. Homer, Endomorphisms of the cohomology rings of finite Grassmann manifolds, Lecture Notes in Math., vol. 657, Springer-Verlag, Berlin and New York, 1978, pp. 179-193.

6. Self-maps of flag manifolds. Trans. Amer. Math. Soc. 267 (1981), 423-434.

7. H. Glover and G. Mislin, On the genus of generalized flag manifolds, Enseign. Math. (2) 27 (1981), 211-219.

8. P. Griffiths and J. Harris, Principles of algebraic geometry, Wiley, New York, 1978.

9. P. Griffiths and J. W. Morgan, Rational homotopy theory and differential forms, Birkhauser, Boston, Mass., 1981.

10. W. V. D. Hodge, The theory and applications of harmonic integrals, 2nd ed., Cambridge Univ. Press, London, 1952.

11. M. Hoffman, Cohomology endomorphisms of complex flag manifolds, Ph. D. Thesis, Massachusetts Institute of Technology, 1981.

12. G. Horrocks, On the relations of S-functions to Schubert varieties, Proc. London. Math. Soc. 7 (1957), 265-280.

13. S. Lefschetz, L'analysis situs et la géométrie algébrique, Gauthier-Villars, Paris, 1924; reprinted in Selected papers, Chelsea, New York, 1971.

14. A. Liulevicius, Equivariant K-theory and homotopy rigidity, Lecture Notes in Math., vol. 788. Springer-Verlag, Berlin and New York, 1980, pp. 340-358.

15. I. Macdonald, Symmetric functions and Hall polynomials, Clarendon Press, Oxford, 1979.

16. L. O’Neill, On the fixed point property for Grassmann manifolds, Ph.D. Thesis, Ohio State University, 1974.

17. R. Stanley, Some combinatorial aspects of the Schubert calculus, Lecture Notes in Math., vol. 597. Springer-Verlag, Berlin and New York, 1977, pp. 217-251.

18. A. Weil, Introduction à l'étude des variétés kählériennes, Hermann, Paris, 1958.

Department of Mathematics and Statistics, Memorial University of Newfoundland, St. John's, Newfoundland, CANADA A1B 3X7

Current address: Department of Mathematics, The Ohio State University, Columbus, Ohio 43210 